\title{
Introduction to the Special Section on Deep Learning in FPGAs
}

The rapid advance of Deep Learning (DL), especially via Deep Neural Networks (DNNs), has been shown to compete with and even exceed human capabilities in tasks such as image recognition, playing complex games, and large-scale information retrieval. However, due to the high computational and power demands of deep neural networks, hardware accelerators are essential to ensure that the computation speed meets the application requirements.

Field-programmable gate arrays (FPGAs) have demonstrated great strength in accelerating deep learning inference with high energy efficiency. To explore the strength of FPGA thoroughly and create a pool of advanced representative research works, we started a call for a special issue of TRETS with the topic of DL on FPGAs. The topics of interest include many different aspects of DL on FPGAs, including compilers, tools, and design methodologies, microarchitectures, cloud deployments, edge or IoT, DNN compression, security, comparison studies, survey studies, and others.

Many people answered this call and submitted their most recent research results. After a subset of the submissions was desk-rejected for quality control purposes, a total of 23 manuscripts went through a full-blown reviewing process. To facilitate a fast, fair, and effective reviewing process for this special issue, we formed a special pool of reviewers who are experts on DL and FPGA topics. After a rigorous reviewing process, eight top-quality papers have been accepted into this special issue so far. The following list shows the title of the paper and the institute(s) of the authors, and highlights the contributions of each article.

High-Efficiency Convolutional Ternary Neural Networks with Custom Adder Trees and Weight Compression

Adrien Prost-Boucle, Alban Bourge, and Frédéric Pétrot

University of Grenoble Alpes and TIMA Laboratory, France

This work presents an architecture dedicated to inference using ternary $\{-1,0,1\}$ weights and activations. This architecture is configurable at design time to provide throughput vs power tradeoffs. It also uses information drawn for the target technologies to adapt to the FPGA resources. This allows to achieve up to $5.2 \mathrm{k}$ fps per Watt for classification on a VC709 board using approximately half of the resources of the FPGA.

FINN-R: An End-to-End Framework for Fast Exploration of Quantized Neural Networks

Michaela Blott, Thomas B. Preußer, Nicholas J. Fraser, Giulio Gambardella, Kenneth O’brien, Yaman Umuroglu, Miriam Leeser, and Kees Vissers

Xilinx Research and Northeastern University, U.S.

This work presents the second generation of the FINN framework, an end-to-end tool which enables design space exploration and automates the creation of fully customized inference engines on FPGAs. Given a neural network description, the tool optimizes for given platforms, design targets and a specific precision. It evaluates a selection of reduced precision neural networks ranging from CIFAR-10 classifiers to YOLO-based object detection on a range of platforms including PYNQ

(c) 2018 Copyright held by the owner/author(s).

1936-7406/2018/12-ART14

https://doi.org/10.1145/3294768

ACM Transactions on Reconfigurable Technology and Systems, Vol. 11, No. 3, Article 14. Pub. date: December 2018. 
and AWSF1, demonstrating throughput at 50 Tera Operations $\left(\mathrm{TO}_{\mathrm{p} / \mathrm{s}}\right)$ on AWSF1 and $5 \mathrm{TOp} / \mathrm{s}$ on embedded devices.

Lightening the Load with Highly Accurate Storage- and Energy-Efficient LightNNs

Ruizhou Ding, Zeye Liu, R. D. (Shawn) Blanton, and Diana Marculescu

Carnegie Mellon University, $P A$

This work proposes a new DNN architecture, LightNN, which replaces the multiplications to one shift or a constrained number of shifts and adds. Theoretical analysis for LightNNs show that their accuracy is maintained, while dramatically reducing storage and energy requirements. For a fixed DNN configuration, LightNNs have better accuracy at a slight energy increase than Binary Neural Network (BNN), yet are more energy efficient with only slightly less accuracy than conventional DNNs. Also, it shows that LightNNs reduce latency and use fewer FPGA resources compared to conventional DNN architectures.

NEURAghe: Exploiting CPU-FPGA Synergies for Efficient and Flexible CNN Inference Acceleration on Zynq SoCs

Paolo Meloni, Alessandro Capotondi, Gianfranco Deriu, Michele Brian, Francesco Conti, Davide Rossi, Luigi Raffo, and Luca Benini

Università di Bologna and Università di Cagliari, Italy

ETH Zurich, Switzerland

This work presents NEURAghe, which leverages the synergistic usage of Zynq ARM cores and of a powerful and flexible Convolution-Specific Processor deployed on the reconfigurable logic. The Convolution-Specific Processor embeds both a convolution engine and a programmable soft core, releasing the ARM processors from most of the supervision duties, so the ARM cores can execute hard-to-accelerate parts of the computational graph. NEURAghe supports end-to-end Convolutional Neural Network (CNN)-based classification with a peak performance of 169 Giga Operations (GOps/s), and an energy efficiency of 17 GOps/W.

Optimizing CNN-based Segmentation with Deeply Customized Convolutional and Deconvolutional Architectures on FPGA

Shuanglong Liu, Hongxiang Fan, Xinyu Niu, Ho-Cheung Ng, Yang Chu, and Wayne Luk Imperial college, United Kingdom

This work proposes and develops deconvolution architecture for efficient FPGA implementation. Memory sharing between the computation modules is proposed for the FPGA-based CNN accelerator as well as for other optimization techniques. A non-linear optimization model is introduced to efficiently explore the design space. Also, a hardware mapping framework is developed to automatically generate the low-latency hardware design for any given $\mathrm{CNN}$ model on the target device. The authors implement designs on Xilinx Zynq ZC706 board and the deconvolution accelerator achieves high performance.

You Can't Improve What You Don't Measure: FPGA vs. ASIC Efficiency Gaps for Convolutional Neural Network Inference

Andrew Boutros, Sadegh Yazdanshenas, and Vaughn Betz

University of Toronto, Canada

This work implements three state-of-the-art computing architectures (CAs) for CNN inference on FPGAs and Application Specific Integrated Circuit (ASIC). By comparing the FPGA and ASIC implementations, it highlights the area and performance costs of programmability to pinpoint the inefficiencies in current FPGA architectures. The authors present detailed performance and 
area gaps between FPGA and ASIC. Based on this study, the authors suggest FPGA architectural changes such as increasing Digital Signal Processing (DSP) block count, enhancing low-precision support in DSP blocks and rethinking the on-chip memories to reduce the programmability gap for DL applications.

\section{ReDCrypt: Real-Time Privacy-Preserving Deep Learning Inference in Clouds Using FPGAs \\ Bita Darvish Rouhani, Siam Umar Hussain, Kristin Lauter, and Farinaz Koushanfar UC San Diego and Microsoft Research}

This work proposes ReDCrypt, the first reconfigurable hardware-accelerated framework that empowers privacy-preserving inference of deep learning models in cloud servers. Unlike prior work, ReDCrypt neither requires to change how AI models are trained nor relies on two non-colluding servers to perform. The privacy-preserving computation in ReDCrypt is executed using Yao's Garbled Circuit protocol. Proof-of-concept evaluations for different DL applications demonstrate up to 57-fold higher throughput per core compared to the best prior solution with no drop in the accuracy.

\section{Instruction Driven Cross-layer CNN Accelerator for Fast Detection on FPGA}

Jincheng Yu, Guangjun Ge, Yiming Hu, Xuefei Ning, Jiantao Qiu, Kaiyuan Guo, Yu Wang, and Huazhong Yang

\section{Tsinghua University, China}

This paper presents an overview of important existing works on neural network inference accelerators using FPGAs. It summarizes various existing software and hardware optimization techniques, providing a rich set of references to help readers understand the key issues, challenges, and solutions for developing high speed and energy efficient FPGA accelerators for deep learning. It also provides guidelines for future studies of this important area.

In summary, the special issue covers topics ranging from promising low-bitwidth quantizationbased design methodologies and architectures, embedded CPU/FPGA co-design, conv/deconv acceleration, FPGA/ASIC comparison, privacy-preserving DL inference in the cloud, to a thorough survey study touching on many proposed existing design and optimization techniques for DL on FPGA. We believe this special issue provides important in-depth knowledge and research results as well as key insights for DL on FPGA, benefits the research community, and would guide the future research for this important area.

We would like to thank all the authors for their great interest, hard work, and distinguished research contributions through submissions to this special issue of TRETS. Due to limited space, we can only accept a very limited number of papers. We also would like to sincerely thank all the reviewers who have dedicated a significant amount of effort to provide high-quality reviews, sometimes a few rounds of reviews for a single paper. The list of reviewers is attached at the end of this editorial to acknowledge their outstanding contributions to make this special issue possible.

Deming Chen, University of Illinois at Urbana-Champaign Andrew Putnam, Microsoft Research

Guest Editors

Steve Wilton, University of British Columbia Editor-in-Chief 